\title{
Modelos de Maturidade da Capacidade SIG: Um Estudo de Caso Utilizando o Modelo GISCMM na Prefeitura Municipal de Porto Alegre/RS
}

\author{
Rodrigo de Marsillac Linn ${ }^{1}$, José Palazzo Moreira de Oliveira ${ }^{2}$ \\ ${ }^{1}$ Secretaria Municipal do Meio Ambiente e da Sustentabilidade, Prefeitura Municipal de Porto \\ Alegre (PMPA) - Porto Alegre - RS - Brazil \\ ${ }^{2}$ Departamento de Informática, Universidade Federal do Rio Grande do Sul (UFRGS) \\ Caixa Postal 15.064 - 91.501-970 - Porto Alegre - RS - Brazil \\ rodrigomlesmurb.prefpoa.com.br, palazzo@inf.ufrgs.br
}

\begin{abstract}
Geographic Information Systems (GIS) is a technology with significant potential for transformation for Governments. Since 1999, the municipal government of Porto Alegre, capital of the State of Rio Grande do Sul, has initiated a series of actions to develop and implement a enterprise GIS. However the imbalance in the development of enterprise GIS is very strong between different institutions within Porto Alegre City Hall (PMPA). In this context, the proposal of this work was that of carrying out a comparative study between three different models of maturity of GIS capacity. This is done in order to identify the most appropriate model for measurement of the GIS maturity level of institutions within the centralized and decentralized administration of Porto Alegre City Hall (PMPA).
\end{abstract}

Resumo. O Sistema de Informação Geográfica (SIG) é uma tecnologia com grande potencial de transformação para os governos. Desde 1999, o governo municipal de Porto Alegre, capital do Estado do Rio Grande do Sul, deu início a uma série de ações para desenvolver e implementar um SIG corporativo. No entanto, há um enorme desequilíbrio no desenvolvimento em SIG entre os órgãos da Prefeitura Municipal de Porto Alegre (PMPA). Nesse contexto, a proposta deste trabalho foi realizar uma análise comparativa entre três modelos de maturidade de capacidade SIG desenvolvidos para organizações públicas com o objetivo de identificar o modelo mais adequado para medir o nível de maturidade SIG dos órgãos da administração centralizada e descentralizada da Prefeitura Municipal de Porto Alegre (PMPA).

\section{Introdução}

O êxodo rural teve como maior efeito a diminuição da população rural e o crescimento da população urbana. Segundo o relatório World Urbanization Prospects (2014) produzido pela Divisão das Nações Unidas para a o Departamento dos Assuntos Econômicos e Sociais (DESA) hoje, no mundo, a população urbana é maior do que população rural. Em 2007, pela primeira vez na história, a população urbana excedeu a população rural, permanecendo predominantemente urbana depois disso.

Ainda segundo o relatório, o processo de urbanização deverá continuar de modo que, até 2050, dois terços da população mundial residirá em território urbano, aproximadamente o inverso da distribuição da população rural-urbana global de meados século XX [UNITED NATIONS, DEPARTMENT OF ECONOMIC AND SOCIAL AFFAIRS, 2014]. 
Com o aumento populacional, o espraiamento do território urbano (urban sprawl)[WILLIAM BURKEY, 2014] e a diversificação das atividades humanas, a gestão das cidades e, especialmente das grandes metrópoles, tornou-se um dos maiores desafios do Século XXI. Planejar e gerir o uso e ocupação do solo, infraestruturas de redes de água e esgoto, iluminação pública, pavimentação, serviços de saúde, educação e segurança, exigem dos gestores e do corpo técnico dos municípios acesso à base de informações governamentais e imensa capacidade de manipular dados. Embora a maioria dos grandes centros urbanos possuam órgãos responsáveis pelo planejamento urbano e pela manutenção dos serviços públicos, muitos carecem de dados e informações para que possam subsidiar a tomada de decisão, possibilitar a transparência das ações e dos atos públicos e disponibilizar à população informações suficientes para exercer ativamente seus direitos de participação democrática nos assuntos da administração municipal.

De outro lado, o surgimento de tecnologias como os Sistemas de Informações Geográficas (SIG) (ou Geographic Information System - GIS) criou enorme possibilidade de adquirir, armazenar, recuperar, transformar e disponibilizar informações espaciais, podendo ser utilizados em estudos relativos ao planejamento urbano, ao meio ambiente, na pesquisa da previsão de determinados fenômenos e no suporte a tomada de decisões [BURROUGH, 1986]. Por essa razão, o SIG é uma tecnologia com grande potencial para transformar os governos, aprimorando os processos de negócios e fornecendo uma plataforma para gerenciar dados espaciais e não espaciais [ALRWAIS, 2016].

A difusão e a utilização do SIG na administração pública iniciaram muito antes nos países desenvolvidos. Segundo Donk e Taylor (2000), o primeiro SIG foi utilizado em 1962, em Ottawa, pelo Departamento Florestal Canadense, para mapear o uso do solo, dados sobre solos, agricultura e vida selvagem. No Brasil, teve início nos anos 80 [CÂMARA, 1996].

Seguindo essa tendência, o governo do município de Porto Alegre, capital do Estado do Rio Grande do Sul, vem adotando, desde 1999, inúmeras iniciativas para desenvolver e implementar um SIG corporativo. Atualmente, uma grande quantidade de informação geográfica é produzida e mantida pelos mais diversos órgãos da administração centralizada e descentralizada do Município e disponibilizada aos técnicos e à sociedade civil através de aplicações WebGIS. Como exemplo, podemos citar a aplicação Declaração Municipal Informativa das Condições Urbanísticas de Ocupação do Solo (DMI) ${ }^{1}$, que integra parte das informações alfanuméricas e geográficas produzidas pelo Município para consulta às informações do regime urbanístico (Densidade, Atividade, Índice de Aproveitamento e Volumetria), alinhamento predial, imóveis tombados, redes de infraestrutura (água, esgoto, energia elétrica e gás) e restrições administrativas (faixas não edificáveis, zonas de proteção a aeródromos e helipontos, áreas de preservação permanente) necessárias para o estudo de viabilidade, elaboração de projetos de edificação e parcelamento do solo, prospecção de áreas para empreendimentos/atividades especificas.

No entanto, a infraestrutura necessária para atender à crescente demanda para produção, manutenção e disponibilização de dados espaciais e serviços de SIG encontra-se subdimensionada. Além disso, há um enorme desequilíbrio no nível de maturidade SIG entre os órgãos da Prefeitura Municipal de Porto Alegre (PMPA), muitos dos processos são ad-hoc e uma grande parcela dos dados não possuem uma estrutura padronizada. Ainda, a falta de coordenação e de indicadores que possam expor as áreas mais sensíveis e mais carentes de SIG dificultam um plano de ação para mitigação ou modernização.

\footnotetext{
${ }^{1}$ http://www2.portoalegre.rs.gov.br/dm/
} 
Nesse contexto, a proposta deste estudo foi realizar uma análise comparativa entre três modelos de avaliação da maturidade da capacidade SIG, desenvolvidos para organizações públicas: o Modelo de Maturidade SIG (GIS Maturity Model), proposto por Jaana Mäkelä (2013), o Modelo de Maturidade de Uso SIG (GIS Usage Maturity Model), proposto por Omer Abdulaziz Alrwais (2016) e o Modelo de Maturidade da Capacidade SIG (GIS Capability Maturity Model - GISCMM), proposto por Greg Babinski (2013), para posteriormente avaliar a maturidade da capacidade SIG da PMPA de forma a diagnosticar as dimensões/áreas mais críticas. Para escolha do modelo utilizou-se como referência as variáveis adotadas no estudo de Khoshgpftar \& Osman (2009).

\section{Modelos de maturidade}

A partir da década de 70, os modelos de maturidade foram reconhecidos como importantes ferramentas de melhoria para as organizações. São ferramentas evolutivas para avaliar e melhorar sistematicamente as capacidades, ou seja, habilidades ou competências, para alcançar a excelência nos processos de negócio [VAN LOOY et al., 2013]. Os modelos de maturidade podem ser utilizados para "avaliar o estado atual da competência, definir um roteiro para melhoria organizacional e avaliar os efeitos do desenvolvimento" [MÄKELÄ, 2012]. Desde então, dezenas de Modelos de Maturidade de Processos de Negócios (Business Process Maturity Models - BPMMs) foram desenvolvidos.

O primeiro modelo de maturidade desenvolvido para Tecnologia da Informação (TI) foi o modelo "estágios de crescimento", criado por Gibson e Nolan, em 1974, e aperfeiçoado em 1979, onde propuseram uma progressão de quatro estágios de despesas com TI que segue uma curva $\mathrm{S}$ desde a iniciação até a integração. O mais influente, o Modelo de Maturidade e de Capacidade (Capability Maturity Model - CMM), foi proposto pelo Software Engineering Institute (SEI), da Carnegie Mellon University em 1989, para avaliar a capacidade dos fabricantes de softwares de concluir com sucesso grandes projetos de desenvolvimento de softwares [BABINSKI, 2011].

Desde então, o conceito de CMM tem sido aplicado à engenharia de sistemas, gerenciamento de projetos, gerenciamento de riscos e serviços de tecnologia da informação. O CMM avalia o nível de maturidade de uma organização com base em como ela executa processos individuais.

Constituídos de vários estágios, os modelos de maturidade descrevem padrões típicos no desenvolvimento de capacidades organizacionais, identificando os domínios nos quais os recursos são relevantes e um modelo de avaliação. Os primeiros (também chamados de critérios ou dimensões) definem o escopo de uma avaliação de maturidade, o último define os níveis de maturidade alcançáveis pelas organizações dentro dos domínios identificados.

É comumente aceito avaliar a maturidade de uma determinada capacidade em cinco níveis possíveis de maturidade, desde inicial / ad hoc, quando a organização está começando a perceber a existência e o potencial de uma capacidade, até a otimização, quando uma capacidade é amplamente disponível na organização, gerida de forma eficaz e revista periodicamente para melhoria [COMUZZI; PATEL, 2016].

Os níveis de maturidade são geralmente definidos como:

- Nível 1: processo ad hoc;

- Nível 2: processo repetível - com base na metodologia anterior bem-sucedida;

- Nível 3: processo definido - processos bem-sucedidos, documentados para orientar o desempenho consistente;

- Nível 4: processo gerenciado - processos documentados medidos e analisados; e, 
- Nível 5: processo otimizado - processos definidos e gerenciados aprimorados por atividades de melhoria contínua de processos.

\subsection{Modelo de Maturidade SIG}

Um modelo de maturidade SIG pode ser utilizado para avaliar a maturidade de uma organização na utilização de dados geográficos, indicando a capacidade e disposição em utilizar esse tipo de informação para alcançar seus objetivos de negócio. Numa organização madura, os dados geográficos são utilizados em todos os processos de negócio, apoiando a execução das prioridades da organização e a tomada de decisões [MARR; BENWELL, 1995].

Apesar de que o uso de dados geográficos e soluções espaciais possam aumentar significativamente a produtividade em muitas organizações, a exploração de todo o potencial ainda está longe de ser alcançada [MÄKELÄ; VANIALA; AHONEN-RAINIO, 2010]. Mesmo que as organizações desejem aumentar a sua capacidade em utilizar esses dados nos seus negócios, nem sempre é claro quais áreas de desenvolvimento devem receber investimentos, neste caso, um modelo de maturidade de capacidade SIG pode ser utilizado como uma ferramenta para avaliar o estado atual da competência e efetuar um avaliação comparativa de desempenho (benchmarking) para definir um roteiro para melhoria organizacional e para avaliar os efeitos do desenvolvimento.

Até 2018, pouco mais de uma dezena de modelos de maturidade SIG foram desenvolvidos por acadêmicos, especialistas, instituições e empresas de consultoria [ALRWAIS et al., 2015]. Não surpreendentemente, a maioria deles foi desenvolvida e aplicada em governos locais, devido ao longo histórico no uso de SIG. Embora o contexto seja semelhante para esses modelos (governo local), eles medem a maturidade SIG a partir de diferentes perspectivas.

\section{Proposta de Análise}

Para a elaboração deste trabalho foram avaliados os modelos desenvolvidos com foco nas organizações públicas e, preferencialmente, com foco nos governos locais. Inicialmente, foram selecionados os três modelos que apresentaram maiores números de citações e maior grau de confiabilidade da instituição geradora.

\subsection{Variáveis analisadas}

Das vinte e sete variáveis propostas inicialmente, no estudo realizado por Khoshgoftar e Osman (2009), quatorze foram selecionadas para serem aplicadas neste trabalho: Data da publicação; Foco; Escopo do modelo; Dimensões; Número de níveis de maturidade; Discreto / Contínuo (em referência ao nível de maturidade); Fatores considerados pelo modelo; Padrão adotado pelo modelo (referem-se a qual a referência o modelo adotou); Considera a estratégia da organização; Dificuldade (complexidade) de Avaliação; Sugere alternativa para melhorias; Documentação disponível para aplicar o modelo; Variáveis e Embasamento.

\section{Resultados da análise}

A partir da análise da matriz comparativa dos modelos de maturidade (Tabela 1) pôde-se observar que as áreas (dimensões) consideradas pelos modelos são muito semelhantes, assim como os níveis de maturidade que foram adotados, exceto para o Modelo de Maturidade de Uso SIG, que possui apenas três níveis (Exploração, Utilização e Corporativo). 
Tabela 1: Matriz comparativa.

\begin{tabular}{|c|c|c|c|}
\hline Variáveis de avaliação & $\begin{array}{c}\text { Modelo de Maturidade SIG (GIS } \\
\text { Maturity Model) }\end{array}$ & $\begin{array}{c}\text { Modelo de Maturidade de Uso } \\
\text { SIG (GIS Usage Maturity } \\
\text { Model) }\end{array}$ & $\begin{array}{c}\text { Modelo de Maturidade de } \\
\text { Capacidade SIG (GIS Capability } \\
\text { Maturity Model) }\end{array}$ \\
\hline 1- Data da publicação & 2012 & 2016 & 2009 (atualizado em 2013) \\
\hline 2- Foco & Organizações públicas & Governo local & Governo local \\
\hline 3- Escopo do modelo & Maturidade em SIG & Maturidade em SIG & Maturidade em SIG \\
\hline 4- Dimensões & $\begin{array}{ll}\text { 1- } & \text { Arquitetura } \\
\text { 2- } & \text { Serviços e Processos } \\
\text { 3- } & \text { Capacidades }\end{array}$ & $\begin{array}{ll}\text { 1- } & \text { Sistema } \\
\text { 2- } & \text { Tarefas } \\
\text { 3- } & \text { Usuários } \\
\text { 4- } & \text { Organização } \\
\text { 5- } & \text { Departamento SIG }\end{array}$ & $\begin{array}{l}\text { 1- Capacidade de Habilitação } \\
\text { 2- Capacidade de Execução }\end{array}$ \\
\hline 5- Número de niveis de & $\begin{array}{l}\text { 1- } \quad \text { Caso Específico } \\
\text { 2- } \text { Administrado Separadamente em } \\
\text { Cada Ramo }\end{array}$ & $\begin{array}{l}\text { 1- Exploração } \\
\text { 2- Utilização }\end{array}$ & $\begin{array}{l}\text { Capacidade de Habilitação } \\
6 \text { Níveis } 1 \text { - } 6\end{array}$ \\
\hline $\begin{array}{l}\text { maturidade: A quantidade de níveis } \\
\text { de maturidade do modelo }\end{array}$ & $\begin{array}{ll}\text { 3- } & \text { Coordenado Concentrado } \\
\text { 4- } & \text { Compreensivelmente Administrado } \\
\text { 5- } & \text { Estrategicamente Otimizado } \\
\text { 6- } & \text { Inovação }\end{array}$ & 3- Corporativo & $\begin{array}{l}\text { Capacidade de Execução } \\
5 \text { Níveis } 1 \text { - } 5 \text { CMM }\end{array}$ \\
\hline $\begin{array}{l}\text { 6- Discreto / Contínuo: Em } \\
\text { referência ao nivel de maturidade }\end{array}$ & Contínuo & Contínuo & Discreto \\
\hline $\begin{array}{l}\text { 7- Fatores considerados pelo } \\
\text { modelo }\end{array}$ & $\begin{array}{l}\text { Infraestrutura, qualidade dos dados, } \\
\text { serviços e processos SIG, capacidade } \\
\text { das equipes e da organização na } \\
\text { utilização de dados espaciais, inovação }\end{array}$ & $\begin{array}{l}\text { Infraestrutura, serviços e processos } \\
\text { SIG, capacidade das equipes e da } \\
\text { organização, foco no uso do SIG, } \\
\text { relação entre SIG e estratégia da } \\
\text { organização, estrutura } \\
\text { organizacional (departamento SIG), } \\
\text { gestão, valor SIG }\end{array}$ & $\begin{array}{l}\text { Infraestrutura; qualidade dos dados, } \\
\text { serviços e processos SIG; } \\
\text { capacidade das equipes e da } \\
\text { organização; relação entre SIG e } \\
\text { estratégia da organização, operação }\end{array}$ \\
\hline $\begin{array}{l}\text { 8- Padrão adotado pelo modelo: } \\
\text { Refere-se a qual a referência o } \\
\text { modelo adotou }\end{array}$ & CMM & CMM & CMM \\
\hline $\begin{array}{l}\text { 9- Considera a estratégia da } \\
\text { organização }\end{array}$ & Sim & Sim & Sim \\
\hline $\begin{array}{l}\text { 10- Dificuldade (complexidade) de } \\
\text { Avaliação }\end{array}$ & Média & Alta & Baixa \\
\hline $\begin{array}{l}\text { 11- Sugere alternativa para } \\
\text { melhorias }\end{array}$ & Não & Não & Não \\
\hline $\begin{array}{l}\text { 12- Documentação disponível para } \\
\text { aplicar o modelo }\end{array}$ & Não & Sim & Sim \\
\hline 13- Variáveis & 15 & 24 & 45 \\
\hline 14- Embasamento & Teórico e prático & Teórico & Prático \\
\hline
\end{tabular}

Sob uma abordagem qualitativa, algumas características foram classificadas como fatores positivos e fatores negativos (Tabela 2 ).

Tabela 2: Fatores positivos e negativos de cada modelo.

\begin{tabular}{|c|c|c|}
\hline & Fatores positivos & Fatores negativos \\
\hline \multirow{4}{*}{$\begin{array}{l}\text { Modelo de Maturidade } \\
\text { SIG (GIS Maturity Model) }\end{array}$} & $\begin{array}{l}\text { Único modelo a considerar inovação como nível de } \\
\text { maturidade. }\end{array}$ & Pouca documentação para aplicar o modelo. \\
\hline & $\begin{array}{l}\text { Foi elaborado através de um amplo debate com } \\
\text { especialistas da área de SIG. }\end{array}$ & $\begin{array}{l}\text { Em algumas situações o estado de um nível mais alto é } \\
\text { atendido sem que o estado do nível mais baixo tenha sido }\end{array}$ \\
\hline & - & Modelo muito enxuto para cobrir todo escopo. \\
\hline & - & Modelo pouco aplicado. \\
\hline \multirow{4}{*}{$\begin{array}{l}\text { Modelo de Maturidade } \\
\text { de Uso SIG (GIS Usage } \\
\text { Maturity Model) }\end{array}$} & $\begin{array}{l}\text { Modelo embasado em extensa revisão bibliográfica. } \\
\text { Extremamente detalhado. }\end{array}$ & $\begin{array}{l}\text { Apenas } 3 \text { níveis de maturidade, o que pode levar a uma } \\
\text { classificação imprecisa. }\end{array}$ \\
\hline & $\begin{array}{l}\text { Relaciona maturidade ao uso do SIG na organização. } \\
\text { Pondera muito o uso do SIG pela organização, o que o } \\
\text { torna diferenciado em relação aos outros modelos. }\end{array}$ & No questionário, cita apenas softwares proprietários da ESRI. \\
\hline & $\begin{array}{l}\text { Profunda análise dos fatores organizacionais, visão da } \\
\text { organização em relação ao SIG. }\end{array}$ & $\begin{array}{l}\text { Questionários mais complexo, exige mais tempo e atenção do } \\
\text { participante. }\end{array}$ \\
\hline & Dimensão dedicada ao departamento/unidade de SIG & Não foi utilizado por organizações públicas. \\
\hline
\end{tabular}




\begin{tabular}{|c|c|c|}
\hline & Fatores positivos & Fatores negativos \\
\hline \multirow{8}{*}{$\begin{array}{l}\text { Modelo de Maturidade } \\
\text { de Capacidade SIG (GIS } \\
\text { Capability Maturity } \\
\text { Model) }\end{array}$} & $\begin{array}{l}\text { Foi elaborado através de um amplo debate com } \\
\text { especialistas da área de SIG. }\end{array}$ & $\begin{array}{l}\text { A opção por apenas duas dimensões. Dimensões específicas } \\
\text { facilitam a identificação das áreas mais frágeis. }\end{array}$ \\
\hline & $\begin{array}{l}\text { Mantido por uma associação (URISA) com foco na gestão } \\
\text { em SIG. Desenvolvimento contínuo. }\end{array}$ & $\begin{array}{l}\text { Algumas variáveis baseiam-se em referências do Federal } \\
\text { Geographic Data Commitee e da URISA, dificultando a sua } \\
\text { aplicação em outros países. }\end{array}$ \\
\hline & Muito bem documentado e abertamente disponibilizado. & - \\
\hline & $\begin{array}{l}\text { Amigável, níveis são semelhantes aos propostos pelo CMM, } \\
\text { paramétrico. }\end{array}$ & - \\
\hline & Avaliação alinhada com o Modelo de Gestão de & \\
\hline & Competência (Geospatial Management Competency & - \\
\hline & Model), também mantido pela URISA. & \\
\hline & Amplamente aplicado em organizações públicas locais. & - \\
\hline
\end{tabular}

Considerando que o Modelo GISCMM, além de ser aberto e bem documentado, apresentou mais pontos favoráveis, foi escolhido para ser aplicado na Prefeitura Municipal de Porto Alegre.

Antes do GISCMM ser aplicado, foram necessárias pequenas alterações para adaptar o formulário a uma avaliação entre secretarias/departamentos da mesma organização, neste caso a PMPA, visto que o formato original do Modelo era para avaliação entre organizações (prefeituras, estados). Desta forma, as questões de número oito (Arquitetura) e doze (Backup dos dados e segurança), da dimensão Capacidade de Habilitação, foram eliminadas pois, atualmente, são planejadas e executadas pela empresa de TI da PMPA, a PROCEMPA. Por fim, a questão de número vinte e dois, da dimensão Capacidade de Execução, também foi excluída por necessidade de atendimento a padrões da URISA e do Instituto de Certificação SIG (GIS Certification Institute).

\section{Resultados}

O formulário do GISCMM foi submetido a todos os órgãos da PMPA que possuíam representação no Grupo de Trabalho de Geoprocessamento. Nove secretarias (SMAMS, SMF, SMC, SMDE, SMSEG, SMIM, SMSURB, SMRI, SMS) ${ }^{2}$ e quatro autarquias/departamentos (DEMHAB, DMAE, DMLU e EPTC) $)^{3}$ responderam ao formulário, totalizando treze órgãos da PMPA.

Os resultados são apresentados através de gráficos de radar (radar chart) por oferecem uma maneira clara de exibir observações quantitativas multivariáveis, em que cada variável é representada por um eixo que inicia no centro de um círculo (correspondente ao Nível 0).

A seguir, são apresentadas as questões que foram submetidas através do formulário e os resultados obtidos pelo GISCMM, exibidos através de gráficos radar e gráficos de coluna.

\section{Capacidade de Habilitacão:}

1.Framework de dados espaciais; 2.Manutenção do framework de dados espaciais; 3.Dados espaciais de negócios; 4.Manutenção dos dados espaciais de negócios; 5.Coordenação dos dados espaciais; 6.Metadados; 7.Banco de dados espacial; 9.Infraestrutura técnica; 10.Plano

2 SMAMS-Secretaria Municipal do Meio Ambiente e da Sustentabilidade, SMF-Secretaria Municipal da Fazenda, SMC- Secretaria Municipal da Cultura, SMDE-Secretaria Municipal de Desenvolvimento Econômico, SMSEG-Secretaria Municipal de Segurança, SMIM-Secretaria Municipal de Infraestrutura e Mobilidade Urbana, SMSURB-Secretaria Municipal de Serviços Urbanos, SMRI-Secretaria Municipal de Relações Institucionais, SMS-Secretaria Municipal de Saúde.

${ }^{3}$ DEMHAB-Departamento Municipal de Habitação, DMAE- Departamento Municipal de Água e Esgotos, DMLU-Departamento Municipal de Limpeza Urbana, EPTC- Empresa Pública de Transporte e Circulação. 
de substituição; 11.Manutenção de softwares SIG; 13.Catálogo de aplicativos SIG; 14.Gerenciamento do catálogo de aplicativos SIG; 15.Organização e Métodos (O\&M) catálogo de aplicações SIG; 16.Gerência profissional em SIG; 17.Staff de profissionais de SIG; 18.Treinamento do staff SIG e desenvolvimento profissional; 19.Estrutura de governança em SIG; 20.SIG está vinculado aos objetivos estratégicos da organização; 21.Orçamento SIG; 22.Financiamento SIG e 23.Plano do financiamento SIG.

\section{Capacidade de Execucão:}

1.Avaliação e desenvolvimento de novos serviços ao cliente; 2.Suporte ao usuário, suporte técnico e treinamento para usuários finais; 3.Monitoramento da entrega de serviços e supervisão; 4.Garantia de qualidade de serviço; 5.Desenvolvimento de aplicações ou metodologia de aquisições; 6.Metodologia de gerenciamento de projetos; 7.Garantia de Qualidade e Controle de Qualidade; 8.Gerenciamento do SIG; 9.Gerenciamento de eventos; 10.Gerenciamento de contratos e fornecedores; 11.Colaboração regional; 12.Desenvolvimento de equipe; 13.Gerenciamento de desempenho; 14.Gestão individual de desempenho do Staff; 15.Monitoramento e Garantia da Satisfação do Cliente; 16.Gerenciamento de alocação de recursos; 17.Compartilhamento de dados SIG; 18.Compartilhamento de licença de software SIG; 19.Interoperabilidade de dados SIG; 20.Gestão de assuntos legais e políticos e 21.Equilibrando a mínima privacidade com o uso máximo de dados.

\subsection{Resultados obtidos na Capacidade de Habilitação}

De forma geral, pôde-se comprovar a partir dos resultados obtidos a baixa maturidade em SIG, da PMPA, e a alta discrepância dos recursos disponíveis, os habilitadores, entre os órgãos da PMPA. Na Figura 1 é demonstrada a alta diferença da capacidade de habilitação entre a SMF (maior desempenho/maturidade) em relação à EPTC e SMSEG (menor desempenho/maturidade).

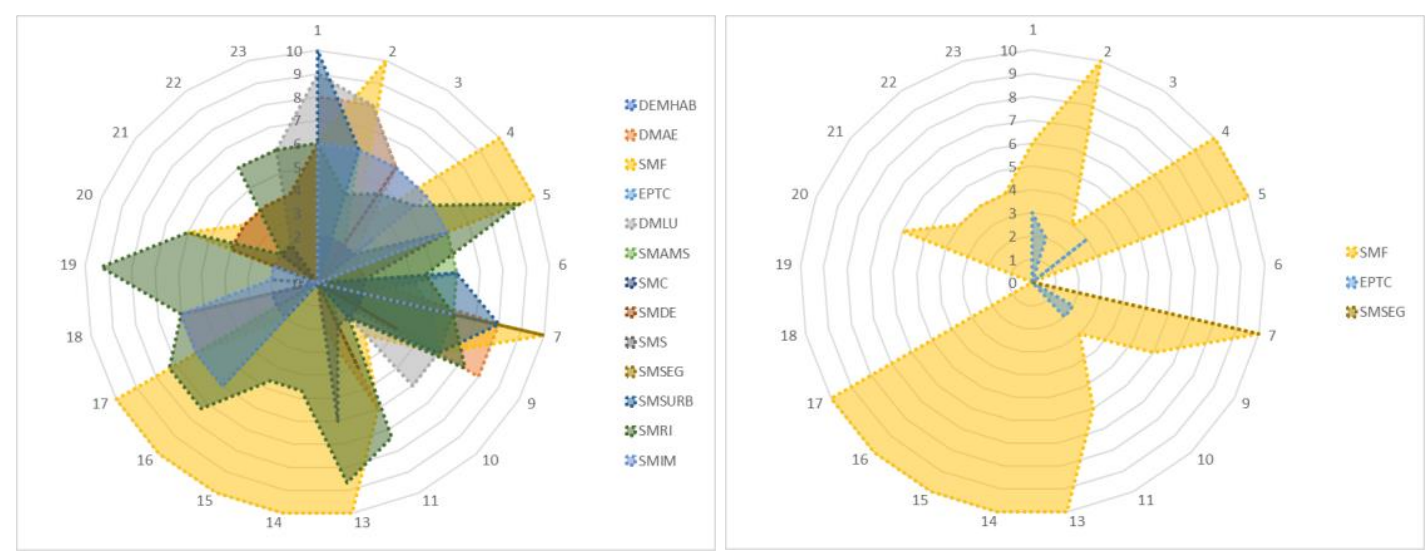

Figura 1: (à esq.) Resultados obtidos pelos 16 órgãos da PMPA em Capacidade de Habilitação. (à dir.) Resultados obtidos pela SMF, EPTC e SMSEG em Capacidade de Habilitação.

Os três piores resultados identificados a partir da média por atividade (Figura 2) para Capacidade de Habilitação, em toda a organização, foram: Estrutura de governança em SIG, Gerenciamento do catálogo de aplicativos SIG e Orçamento SIG. 

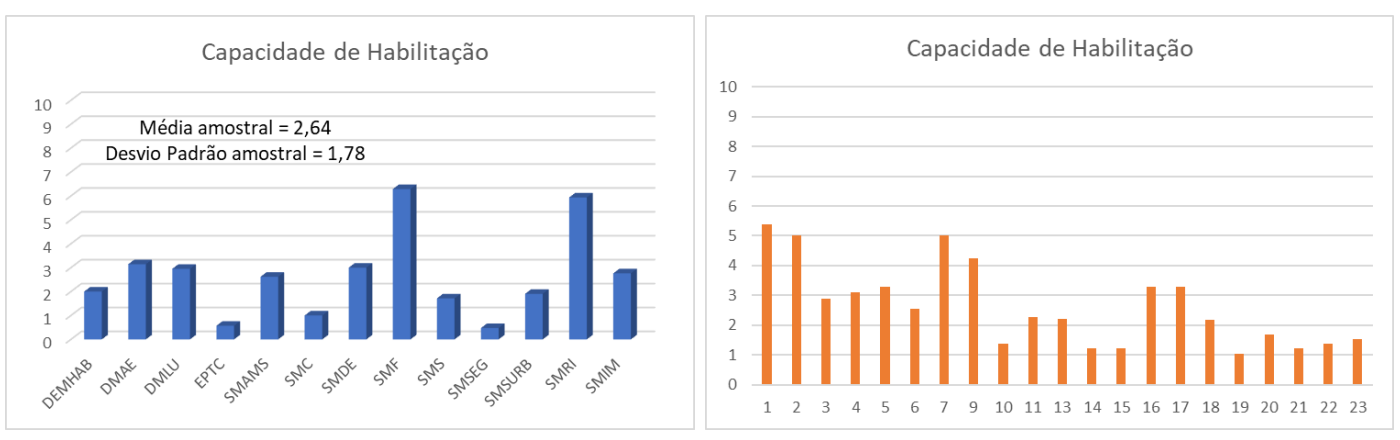

Figura 2: (à esq.) Média final obtida por cada órgão e (à dir.) média por atividade.

\subsection{Resultados obtidos na Capacidade de Execução}

Da mesma forma que a capacidade de habilitação, pôde-se comprovar a alta discrepância na capacidade de execução SIG da PMPA. Na Figura 3 é demonstrada a grande diferença da capacidade de execução entre a SMF (maior desempenho/maturidade) em relação à EPTC e SMSEG (menor desempenho/maturidade).

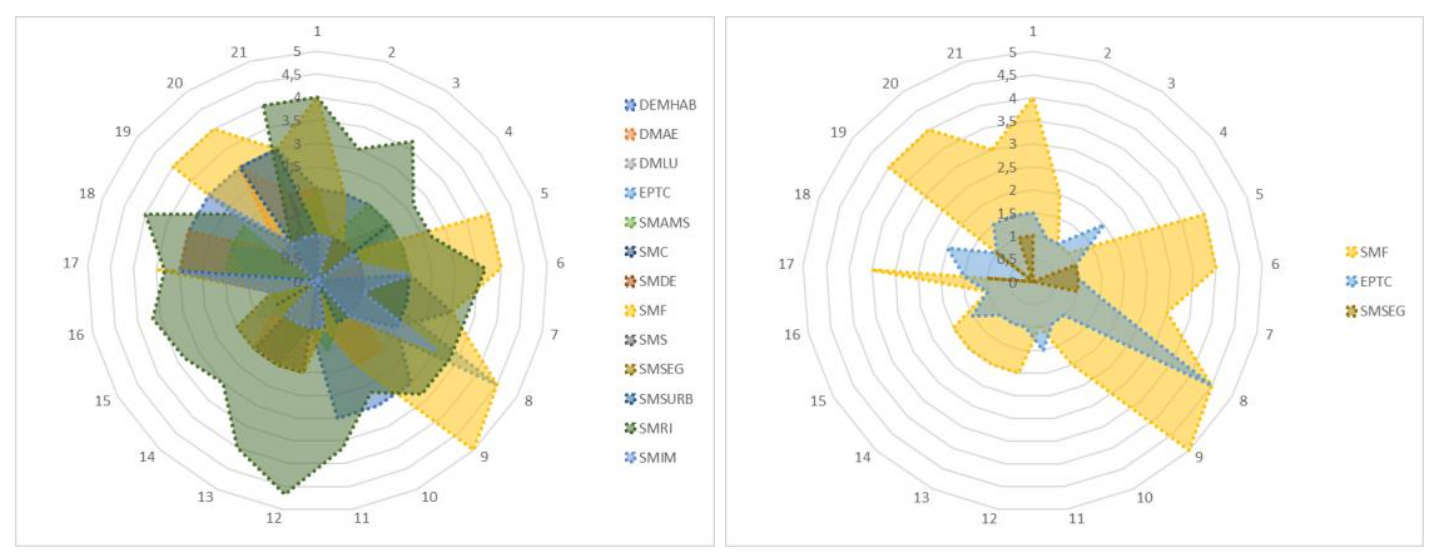

Figura 3: (à esq.) Resultados obtidos pelos 16 órgãos da PMPA em Capacidade de Execução. (à dir.) Resultados obtidos pela SMF, EPTC e SMSEG em Capacidade de Execução.

Os três piores resultados identificados a partir da média por atividade (Figura 4) para Capacidade de Execução foram: Gerenciamento de alocação de recursos, Monitoramento e Garantia da Satisfação do Cliente e Colaboração regional.
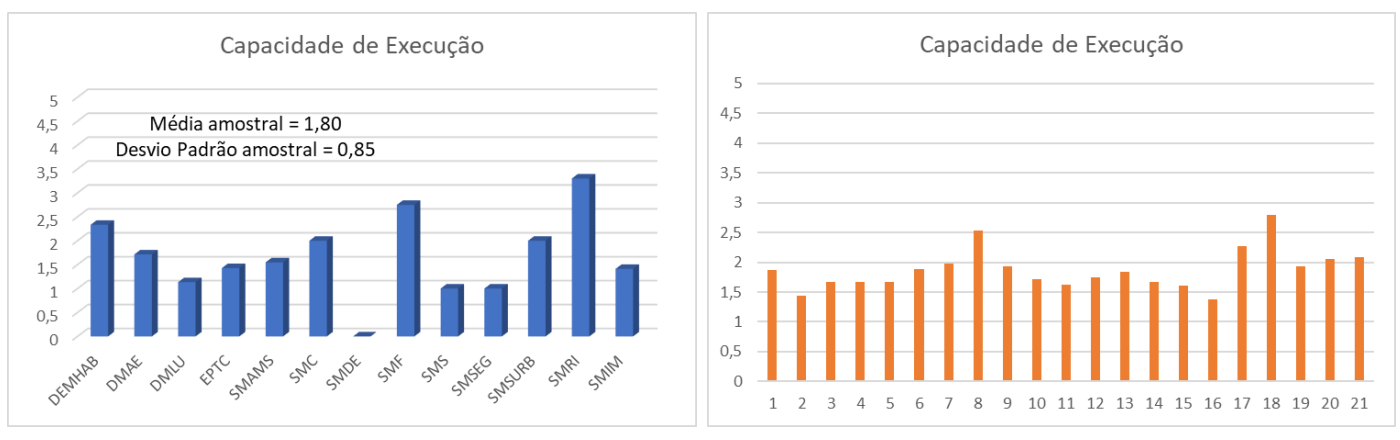

Figura 4: (à esq.) Média final obtida por cada órgão e (à dir.) média por atividade. 


\section{Conclusões}

A partir dos resultados obtidos e da experiência adquirida na aplicação do Modelo GISCMM, pôde-se concluir que:

1. De fato, o nível de maturidade SIG dos órgãos da PMPA é consideravelmente baixo, exceto para SMF;

2. Os órgãos que, sabidamente, possuem o SIG incorporado aos objetivos estratégicos da organização, tendem a possuir maior nível de maturidade, pois acabam investindo mais recursos o que eleva o nível de maturidade. Observa-se esta situação na SMF, SMRI e DMAE;

3. Com base no conhecimento sobre a PMPA, o Modelo apresentou resultados extremamente coerentes, apenas algumas pequenas distorções foram provocadas em razão da falta de compreensão do que estava sendo solicitado no formulário. Constatou-se ser importante realizar um nivelamento com os participantes antes de aplicar o formulário para explicitar o escopo de cada questão;

4. Se a organização não possui um bom nível maturidade nas variáveis 19 - Estrutura de governança em SIG, 20 - SIG estar vinculado aos objetivos estratégicos da organização e 21 - Orçamento SIG, que são "habilitadoras", provavelmente terá uma maturidade global muito baixa, pois estas são as premissas elementares para o desenvolvimento em SIG;

5. O GISCMM, assim como os outros modelos avaliados neste trabalho, foram elaborados para avaliação entre organizações públicas (cidades, estados), em razão do que foi necessário adequar o Modelo para ser aplicado dentro de uma organização, neste caso, a PMPA, que possui uma empresa de TI (PROCEMPA) responsável por toda a infraestrutura (banco de dados geográfico (BDG), arquitetura, backup). Sugere-se que novos estudos sejam realizados com o objetivo de desenvolver um Modelo para este tipo de cenário;

6. A aplicação do GISCMM foi razoavelmente simples e o modelo, fácil de ser compreendido. É, sem dúvida, uma excelente ferramenta de avaliação, resultando em informação confiável para subsidiar a gestão em SIG. Espera-se, com isso, promover novos ciclos de avaliações, a fim de se verificar a evolução/aderência desta tecnologia/cultura dentro da PMPA; e,

7. Sugere-se que os demais modelos avaliados neste trabalho sejam futuramente aplicados, visto que possuem um conteúdo muito rico, robusto e de grande valor.

\section{Referências}

Alrwais, Omer et al. Evaluating Local Government GIS: A New Maturity Model Evaluating Local Government Usage of GIS: A New Maturity Model. In: PRE-ICIS WORKSHOP ON LOCATIONAL ANALYTICS AND BIG DATA 2015, Fort Worth. Anais... Fort Worth

Alrwais, Omer Abdulaziz. Towards a New GIS Maturity Model: An Organizational Usage Perspective. 2016. Claremont Graduate University, [s. 1.], 2016. Disponível em: $<$ http://scholarship.claremont.edu/cgu_etd/100>

Babinski, Greg. Managing GIS. ArcNews, [s. 1.], p. 1-8, 2011. Disponível em: $<$ http://www.esri.com/news/arcnews/winter1011articles/urisa-proposes.html $>$. Acesso em: 18 jul. 2018. 
Babinski, Greg. GIS Capability Maturity Model. Des Plains, IL. Disponível em: $<$ http://www.urisa.org/clientuploads/directory/GMI/GISCMM-Final201309(Endorsed for Publication).pdf $>$. Acesso em: 9 ago. 2018a.

Burrough, Peter Alan. Principles of geographical information systems for land resources assessment. In: JOURNAL OF QUATERNARY SCIENCE. [s.1.] : Oxford University, 1986. p. 193.

Câmara, Gilberto. Desenvolvimento de Sistemas de Informação Geográfica no Brasil: Problemas e Desafios. In: 1996, Rio de Janeiro. Anais... Rio de Janeiro Disponível em: $<$ http://www.dpi.inpe.br/gilberto/present/segeo.html $>$. Acesso em: 23 ago. 2018.

Comuzzi, Marco; PATEL, Anit. How organisations leverage Big Data: a maturity model. Industrial Management \& Data Systems, [s. 1.], v. 116, n. 8, p. 1468-1492, 2016. Disponível em: <http://www.emeraldinsight.com/doi/10.1108/IMDS-12-2015-0495>

Gibson, Cyrus F.; NOLAN, Richard L. Managing the four stages of EDP growth. Harvard Business Review, [s. 1.], v. 52, n. February, p. 76-88, 1974. Disponível em: $<$ https://hbr.org/1974/01/managing-the-four-stages-of-edp-growth $>$

Khoshgoftar, Mohammad; OSMAN, Omar. Comparison of Maturity Models. In: IEEE INTERNATIONAL CONFERENCE ON COMPUTER SCIENCE AND INFORMATION TECHNOLOGY 2009, Beijing, China. Anais... Beijing, China: IEEE, 2009. Disponível em:

$<$ https://ieeexplore-ieeeorg.ez45.periodicos.capes.gov.br/abstract/document/5234402/>

Mäkelä, Jaana. Model for Assessing GIS Maturity of an Organization. In: RAJABIFARD, Abbas; COLEMANN, David (Eds.). Spatially Enabling Government, Industry and Citizens. Needham: GSDI Association Press, 2012. p. 277.

Mäkelä, Jaana. Customizing a maturity model for the evaluation of the development of shared situational awareness and the utilization of spatial information. 2013. Aalto University, [s. 2013. 1.], Disponível em: $<$ https://aaltodoc.aalto.fi/handle/123456789/11312>

Mäkelä, Jaana; VANIALA, Riitta; AHONEN-RAINIO, Paula. Competence management within organisations as an approach to enhancing GIS maturity. International Journal of Spatial Data Infrastructures Research, [s. 1.], v. 5, n. 5, p. 267-285, 2010. Disponível em: <http://ijsdir.jrc.ec.europa.eu/index.php/ijsdir/article/view/134/259>. Acesso em: 18 jul. 2018.

Marr, Andrew J.; BENWELL, George L. Local Government GIS in New Zealand Since 1989 The Information Science Discussion Paper Series. Dunedin. Disponível em: $<$ https://ourarchive.otago.ac.nz/bitstream/handle/10523/854/dp1995-13online.pdf? sequence=3\&isAllowed=y> . Acesso em: 30 jul. 2018.

United Nations, Department of Economic and Social Affairs, Population Department. World Urbanization Prospects. United Nations, [s. 1.], v. 12, p. 32, 2014.

Van Looy, Amy et al. Choosing the right business process maturity model. Information and Management, [s. 1.], v. 50, n. 7, p. 466-488, 2013. Disponível em: $<$ http://dx.doi.org/10.1016/j.im.2013.06.002>

William Burkey. Urban Sprawl. 2014. Disponível em: <https://academic-ebbritannica.ez45.periodicos.capes.gov.br/levels/collegiate/article/urban-sprawl/475347>. Acesso em: 14 ago. 2018. 